AIP Applied Physics

\title{
Role of dynamic polar nanoregions in heterovalent perovskite relaxor: Inelastic light scattering study of ferroelectric $\mathrm{Ti}$ rich $\mathrm{Pb}(\mathrm{Zn} 1 / 3 \mathrm{Nb} 2 / 3) \mathrm{O} 3-$ $\mathrm{PbTiO3}$
}

Md. Saidul Islam, Shinya Tsukada, Wenzhi Chen, Zuo-Guang Ye, and Seiji Kojima

Citation: J. Appl. Phys. 112, 114106 (2012); doi: 10.1063/1.4768278

View online: http://dx.doi.org/10.1063/1.4768278

View Table of Contents: http://jap.aip.org/resource/1/JAPIAU/v112/i11

Published by the American Institute of Physics.

\section{Related Articles}

Quenching-induced circumvention of integrated aging effect of relaxor lead lanthanum zirconate titanate and (Bi1/2Na1/2)TiO3-BaTiO3

Appl. Phys. Lett. 102, 032901 (2013)

Relaxor behavior of ferroelectric $\mathrm{Ca} 0.22 \mathrm{Sr} 0.12 \mathrm{Ba} 0.66 \mathrm{Nb} 2 \mathrm{O} 6$

Appl. Phys. Lett. 102, 022903 (2013)

Structural diversity of the $(\mathrm{Na} 1-\mathrm{xKx}) 0.5 \mathrm{Bi0} .5 \mathrm{TiO} 3$ perovskite at the morphotropic phase boundary

J. Appl. Phys. 113, 024106 (2013)

Composition and orientation dependence of high electric-field-induced strain in

$\mathrm{Pb}(\operatorname{In} 1 / 2 \mathrm{Nb} 1 / 2) \mathrm{O} 3-\mathrm{Pb}(\mathrm{Mg} 1 / 3 \mathrm{Nb} 2 / 3) \mathrm{O} 3-\mathrm{PbTiO} 3$ single crystals

J. Appl. Phys. 112, 126102 (2012)

Influence of electric field on local phase transformations in relaxor ferroelectrics $\mathrm{PbSc} 0.5 \mathrm{Ta} 0.5 \mathrm{O} 3$ and

$\mathrm{Pb} 0.78 \mathrm{Ba} 0.22 \mathrm{Sc} 0.5 \mathrm{Ta} 0.5 \mathrm{O} 3$

J. Appl. Phys. 112, $124111(2012)$

\section{Additional information on J. Appl. Phys.}

Journal Homepage: http://jap.aip.org/

Journal Information: http://jap.aip.org/about/about_the_journal

Top downloads: http://jap.aip.org/features/most_downloaded

Information for Authors: http://jap.aip.org/authors

\section{ADVERTISEMENT}

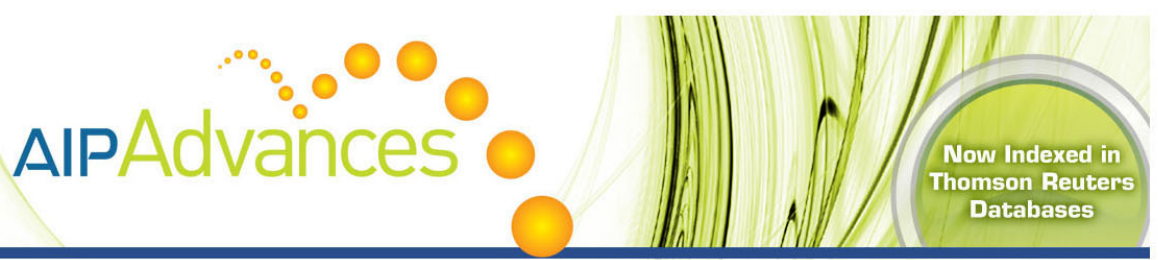

\section{Explore AIP's open access journal: •Rapid publication \\ - Article-level metrics \\ Post-publication rating and commenting}




\title{
Role of dynamic polar nanoregions in heterovalent perovskite relaxor: Inelastic light scattering study of ferroelectric Ti rich $\mathrm{Pb}\left(\mathrm{Zn}_{1 / 3} \mathrm{Nb}_{2 / 3}\right) \mathrm{O}_{3}-\mathrm{PbTiO}_{3}$
}

\author{
Md. Saidul Islam, ${ }^{1,2, a)}$ Shinya Tsukada, ${ }^{1,3}$ Wenzhi Chen, ${ }^{4}$ Zuo-Guang Ye, ${ }^{4}$ and Seiji Kojima ${ }^{1, b)}$ \\ ${ }^{1}$ Graduate School of Pure and Applied Sciences, University of Tsukuba, Tsukuba, Ibaraki 305-8573, Japan \\ ${ }^{2}$ Department of Materials Science and Engineering, University of Rajshai, Rajshai-6205, Bangladesh \\ ${ }^{3}$ Faculty of Education, Shimane University, Matsue city, Shimane 609-8504, Japan \\ ${ }^{4}$ Department of Chemistry and 4D LABS, Simon Fraser University, Burnaby, British Columbia V5A 1S6, \\ Canada
}

(Received 23 July 2012; accepted 1 November 2012; published online 4 December 2012)

\begin{abstract}
The role of dynamic polar nanoregions (PNRs) of $(1-x) \mathrm{Pb}\left(\mathrm{Zn}_{1 / 3} \mathrm{Nb}_{2 / 3}\right) \mathrm{O}_{3}-x \mathrm{PbTiO}_{3}(\mathrm{PZN}-100 x \mathrm{PT})$ single crystals with the PT content $x=0.15$ (i.e., higher than above the morphotropic phase boundary composition $x \sim 0.09$ ) has been investigated using inelastic light scattering. The remarkable anomaly of the longitudinal acoustic mode is clearly observed in the vicinity of the cubic-tetragonal phase transition temperature at the $T_{\mathrm{C}-\mathrm{T}}=481 \mathrm{~K}$. A broad central peak $(\mathrm{CP})$ appears below the Burns temperature $T_{\mathrm{B}} \sim 700 \mathrm{~K}$. Upon cooling, the relaxation time determined from the $\mathrm{CP}$ width clearly shows a critical slowing down when approaching $T_{C-T}$ in contrast with the suppressed slowing down previously observed in PZN-7PT [S. Tsukada and S. Kojima, Phys. Rev. B 78, 144106 (2008)]. These facts are due to the existence of dynamic PNRs and the disappearance of chemically ordered regions resulting from a higher PT content in the solid solution. In the cubic phase, the local symmetry breaking caused by the PNRs was observed by Raman scattering. The initial size of dynamic PNRs is about $3 \mathrm{~nm}$ just below $T_{\mathrm{B}}$, and it increases significantly upon cooling below the intermediate temperature $T^{*} \sim 600 \mathrm{~K}$, reaching $10 \mathrm{~nm}$ down to $T_{C-T}$. (C) 2012 American Institute of Physics. [http://dx.doi.org/10.1063/1.4768278]
\end{abstract}

\section{INTRODUCTION}

$\mathrm{Pb}$-based perovskite relaxors, represented by $\mathrm{Pb}\left(\mathrm{Mg}_{1 / 3}\right.$ $\left.\mathrm{Nb}_{2 / 3}\right) \mathrm{O}_{3}(\mathrm{PMN})$ and $\mathrm{Pb}\left(\mathrm{Zn}_{1 / 3} \mathrm{Nb}_{2 / 3}\right) \mathrm{O}_{3}(\mathrm{PZN})$, exhibit a broad frequency-dependent dielectric peak and Vogel-Fulcher freezing. They have attracted a great deal of research work in the last two decades in their peculiar nonergodic behaviors related to the disordered occupancy of heterovalent cations on the perovskite $B$ sites. The colossal piezoelectric response discovered in the relaxor- $\mathrm{PbTiO}_{3}$ solid solutions has been extensively studied by neutron, x-ray, and inelastic light scattering, and electron diffraction, etc., however, their structure and dynamics remain to be a fascinating puzzle, especially in a paraelectric phase with high symmetry. ${ }^{1-3}$ The solid solution of $(1-x) \mathrm{Pb}\left(\mathrm{Zn}_{1 / 3} \mathrm{Nb}_{2 / 3}\right) \mathrm{O}_{3}-x \mathrm{PbTiO}_{3}(\mathrm{PZN}-100 x \mathrm{PT})$ is one of the relaxor-based ferroelectrics (RFEs) exhibiting a typical relaxor behavior with a diffuse and dispersive dielectric permittivity peak for low PT content, and a morphotropic phase boundary (MPB) where the rhombohedral $(R 3 m)$ and the tetragonal $(P 4 \mathrm{~mm})$ phases coexists for the composition range $x=0.08 \sim 0.10$ (at room temperature). ${ }^{4,5}$ The high piezoresponse of PZN- $x$ PT near the MPB has recently been attributed to the intermediate monoclinic/orthorhombic phases that could facilitate polarization rotation in the symmetry plane. ${ }^{6}$ As the PT content increases from zero towards the MPB, PZN- $x$ PT shows different phase sequences upon cooling. The symmetry and the phase transition anomalies of PZN- $x$ PT near MPB were found to be much more complex than previ-

\footnotetext{
a)sayeed_mse@ru.ac.bd.

b)kojima@ims.tsukuba.ac.jp.
}

ously imagined and monoclinic and/or orthorhombic symmetries appear with and/or without biasing electric field depending on the PT content. ${ }^{7,8}$ However, the exact crystal symmetry, the elastic properties, and the dynamical features of the phase transitions in PZN- $x$ PT over wide composition and temperature ranges still remain unclear, as attested to by a number of controversial results. ${ }^{9-11}$

The compositional disorder, i.e., the disorder in the arrangement of different ions on a crystallographically equivalent site, is a common structural feature of RFEs. The chemically ordered nanoregions (CORs) are formed when the two kinds of different cations with heterovalence states (e.g., $\mathrm{Zn}^{2+}$ and $\mathrm{Nb}^{5+}$ ) occupy the perovskite $B$ sites alternatively. ${ }^{2}$ It is commonly agreed that the polar nanoregions (PNRs) and CORs that are inherently present give rise to unique physical properties of RFEs. ${ }^{12}$ Upon cooling, the dynamic PNRs start to appear at the so called Burns temperature, $T_{\mathrm{B}} \cdot{ }^{13}$ Recently, an intermediate temperature, $T^{*}$, was suggested to exist between $T_{\mathrm{B}}$ and the temperature of maximum dielectric constant, $T_{\mathrm{m}}$. At $T_{\mathrm{B}}$, dynamic PNRs are formed due to the short-lived correlation between the off centered ions, whereas at $T^{*}$, the dynamic PNRs transfer to a static with a rapid growth in size owing to the long-lived correlation between the atomic displacements of off centered ions. ${ }^{14}$ The microscopic mechanism underlying the appearance, growth, dynamics, and length scale of PNRs has not been well understood yet. Another important question on PNRs in the PZN-PT solid solutions is about the upper limit of the PT content at which the PNRs persist. In PMN- $x$ PT, the elastic anomaly owing to the interaction between the longitudinal acoustic (LA) mode and dynamic PNRs was still 
observed in PMN-55PT, i.e., with a PT content beyond the MPB composition. ${ }^{15}$

Recent report on the acoustic emission (AE) of Pb-based relaxors at $T^{*} \sim 500 \mathrm{~K}$ suggests that the martensitic transformation of PNRs generates AE bursts that were detectable by the ultrasonic transducer in the kilohertz range. ${ }^{14}$ While the Brillouin scattering technique probes, the acoustic modes in the gigahertz range with a wavelength much shorter than that of the standard ultrasonic detection. Therefore, the temperature dependence of the local polarization fluctuations of PNRs can be detected with a high sensitivity by the broadband Brillouin scattering technique in the frequency range from 1 to $1000 \mathrm{GHz} .{ }^{16} \mathrm{Up}$ to the present, Brillouin scattering studies have been performed on PZN ${ }^{17,18}$ and the PZN- $x$ PT solid solutions, i.e., PZN-4.5PT crystals, ${ }^{9,19}$ PZN-7PT, ${ }^{20,21}$ PZN-9PT, ${ }^{22-24}$ and PZN-20PT. ${ }^{25}$ The compositions with PT content smaller than $x=0.09$ exhibit relaxor behavior with a diffuse and dispersive permittivity maximum and transforms the relaxor state into a stable ferroelectric phase with rhombohedral symmetry at low temperatures. In contrast, PZN20PT undergoes a first-order ferroelectric phase transition from the cubic paraelectric phase to the tetragonal ferroelectric phase at $T_{\mathrm{C}-\mathrm{T}}=528 \mathrm{~K}$ without diffuse nature and does not enter any rhombohedral ferroelectric phase upon further cooling. ${ }^{26}$ In this letter, we report the Brillouin scattering study of PZN-15PT single crystals which are expected to bridge the gap of physical properties between PZN-9PT and PZN-20PT.

\section{EXPERIMENTAL}

PZN-15PT single crystals were grown by the high temperature solution method as described in Ref. 27. A microBrillouin scattering system with a $3+3$ pass Sandercocktype tandem Fabry-Perot interferometer (FPI) was used to measure the Brillouin spectra at a backward scattering geometry with free spectral ranges 75 and $300 \mathrm{GHz}$. An optical microscope (OLYMPUS BX 41) was associated with the FPI to achieve a fine focal region. A green YAG laser with a wavelength of $532 \mathrm{~nm}$ and a power of $50 \mathrm{~mW}$ was used to excite the sample. A conventional photon counting system and a multi-channel analyzer were employed to detect and average the signals. Raman scattering was measured by a green YAG laser with a wavelength of $532 \mathrm{~nm}$ and a triplegrating spectrometer with additive dispersion (Horiba-JY, T64000). The temperature of the sample was controlled by a heating/cooling stage (Linkam, THMS600) from $350 \mathrm{~K}$ to $830 \mathrm{~K}$ with $\pm 0.1 \mathrm{~K}$ accuracy.

\section{RESULTS AND DISCUSSION}

\section{A. Elastic anomaly}

The temperature dependence of Brillouin scattering spectra was measured in a wide temperature range to investigate the elastic anomaly and the precursor dynamical behavior in the cubic phase. Figures 1(a) and 1(b) show the Brillouin scattering spectra at selected temperatures in broad and narrow frequency ranges, respectively. The spectra of Fig. 1(a) contain a strong doublet of the LA mode around $40 \mathrm{GHz}$ and a broad central peak $(\mathrm{CP})$ around $0 \mathrm{GHz}$. The absence of transverse acoustic (TA) mode at all measurement temperatures demonstrates that the average crystal symmetry is cubic above $T_{\mathrm{C}}$ and tetragonal below $T_{\mathrm{C}}$. The measured Brillouin doublets were fitted by a Lorentzian function convoluted by the Gaussian instrumental function to obtain the Brillouin frequency shift $\left(\nu_{\mathrm{LA}}\right)$ and the full-width at half maximum (FWHM or $\left.\Gamma_{\mathrm{LA}}\right)$. The temperature dependences of $\nu_{\mathrm{LA}}$ related to the elastic constant $C_{11}$ and $\Gamma_{\mathrm{LA}}$ are plotted in Fig. 2. In both heating and cooling processes, a significant softening of the LA mode and a clear anomaly corresponding to the cubic-tetragonal phase transition are observed at $481 \mathrm{~K}\left(T_{C-T}\right)$, which can be attributed to the interaction between the LA mode and the polarization flipping inside the dynamic PNRs. ${ }^{16}$ The observed anomalous temperature dependence is similar to that of PMN55PT with no COR, ${ }^{15,28}$ while such a sharp elastic anomaly is uncharacteristic in typical RFEs such as PMN, PZN with CORs. In addition, $\nu_{\mathrm{LA}}$ shows a mild softening below $T_{\mathrm{B}} \sim 700 \mathrm{~K}$, demonstrating that only normal anharmonic effects dominate in the lattice dynamics above $T_{\mathrm{B}} \cdot{ }^{14}$ On cooling from $T_{\mathrm{B}}$, a remarkable softening of $\nu_{\mathrm{LA}}$ is observed near $T_{C-T}$. The temperature dependence of $\Gamma_{\mathrm{LA}}$, which is related to the acoustic damping of the LA mode, shows the sharp increase near $T_{C-T}$, suggesting the increase of scattering of the LA mode by the growth of dynamic PNRs. ${ }^{15,16}$

\section{B. Local symmetry and PNR}

It is known that Raman scattering is very sensitive to the local symmetry breaking in a cubic phase. ${ }^{26,29}$ The temperature dependence of Raman scattering was measured and shown in Fig. 3. In the cubic phase with space group $P m \overline{3} m$, optical modes have $\mathrm{T}_{1 \mathrm{u}}$ and $\mathrm{T}_{2 \mathrm{u}}$ symmetry, which are Raman
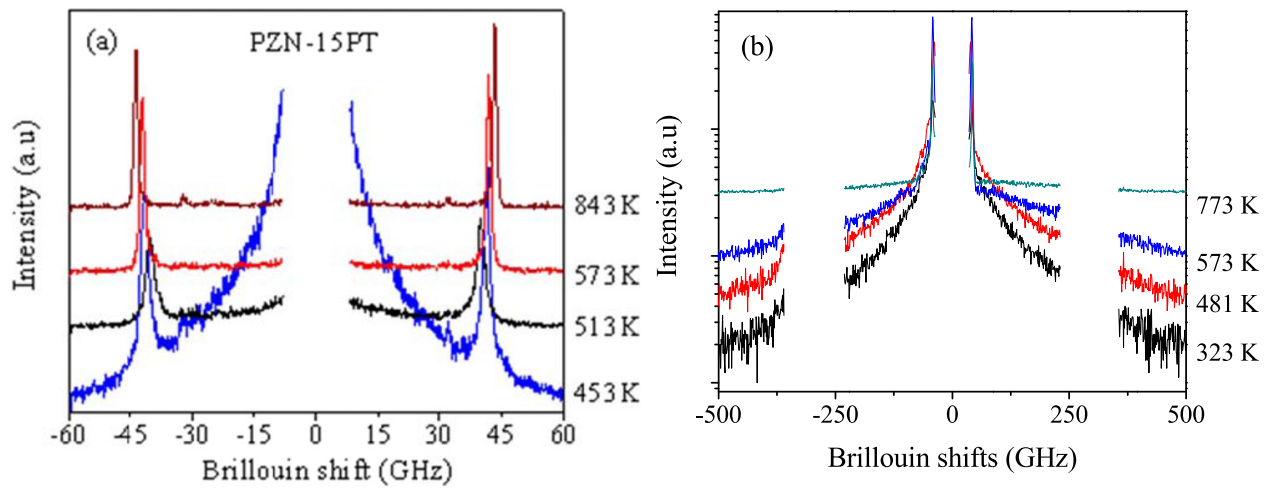

FIG. 1. Brillouin scattering spectra of a PZN-15PT single crystal at selected temperatures in (a) a narrow and (b) a wide frequency ranges for the observation of the LA mode and the $\mathrm{CP}$, respectively. 


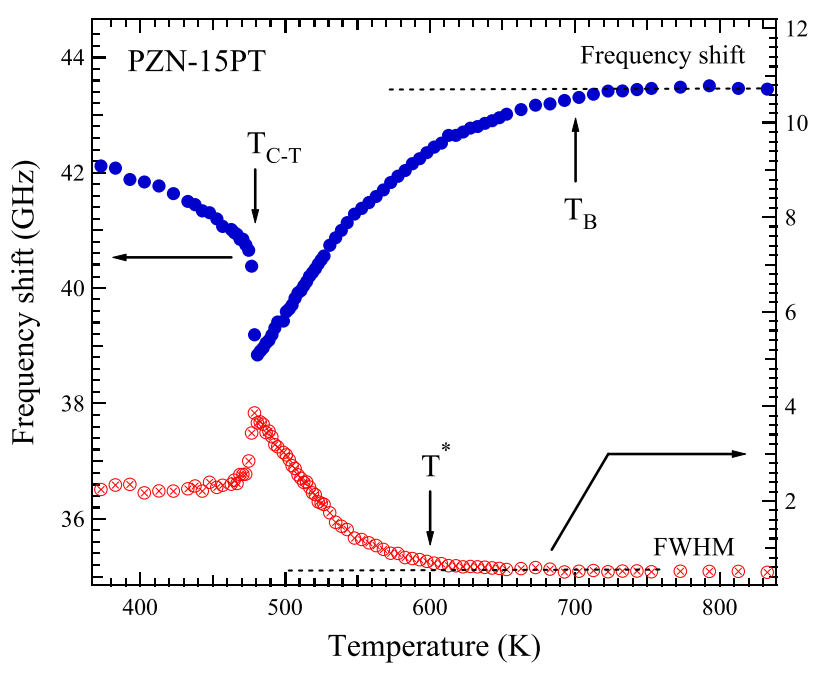

FIG. 2. Temperature dependence of the Brillouin shift and the FWHM of the LA mode of a PZN-15PT single crystal.

inactive. Nevertheless, the Raman spectra (Fig. 3) show the forbidden optical bands arising from the local breaking of the cubic symmetry in PNRs by the existence of local polarization which destroys the center of symmetry. This result is in agreement with inelastic neutron scattering study of PZN15PT which shows the existence of PNRs by the observation of "waterfall" effect. ${ }^{30}$

Raman scattering also provides some important information on the existence of the soft optic mode. The temperature dependence of the Raman spectra shows the lowest frequency optic mode at about $50 \mathrm{~cm}^{-1}$, which is attributed to the positional disorder of lead ions, and this mode does not show any softening, which is the same as the case of PZN-9PT. ${ }^{29}$ Although $\mathrm{PbTiO}_{3}$ shows typical optic soft modes with $E$ symmetry, ${ }^{31}$ PZN-15PT has no displacive nature at the zone center. Since there is no soft mode, the origin of intense $\mathrm{CP}$ appearing below the $T_{\mathrm{B}}$, as shown in Fig. 1(a), cannot be the tail of the overdamped soft optic mode but the relaxation mode instead. Therefore, we have analyzed the $\mathrm{CP}$ in the Brillouin scattering spectra to clarify the critical

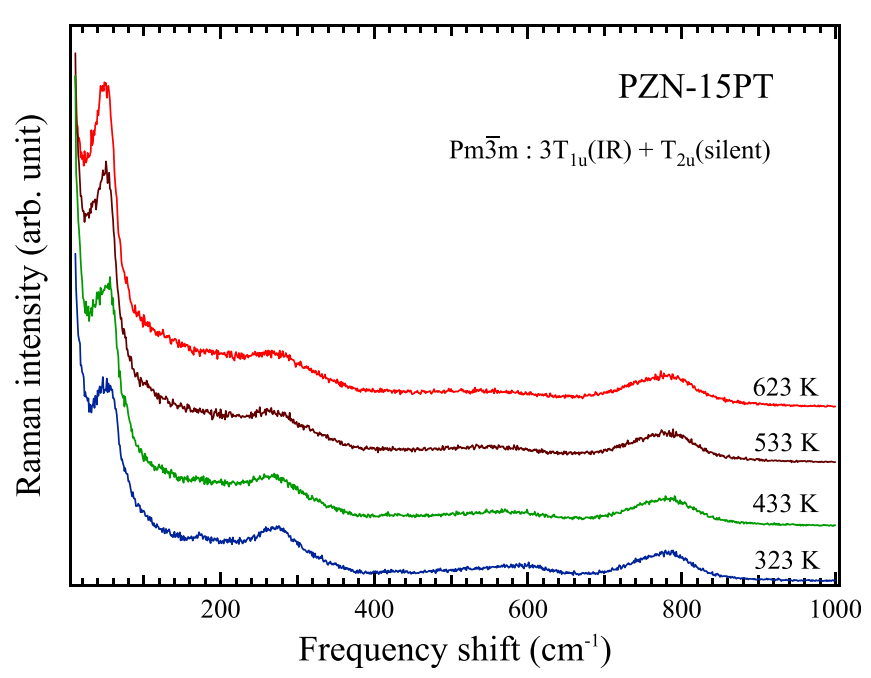

FIG. 3. Temperature dependence of Raman scattering spectra of a PZN15PT single crystal.

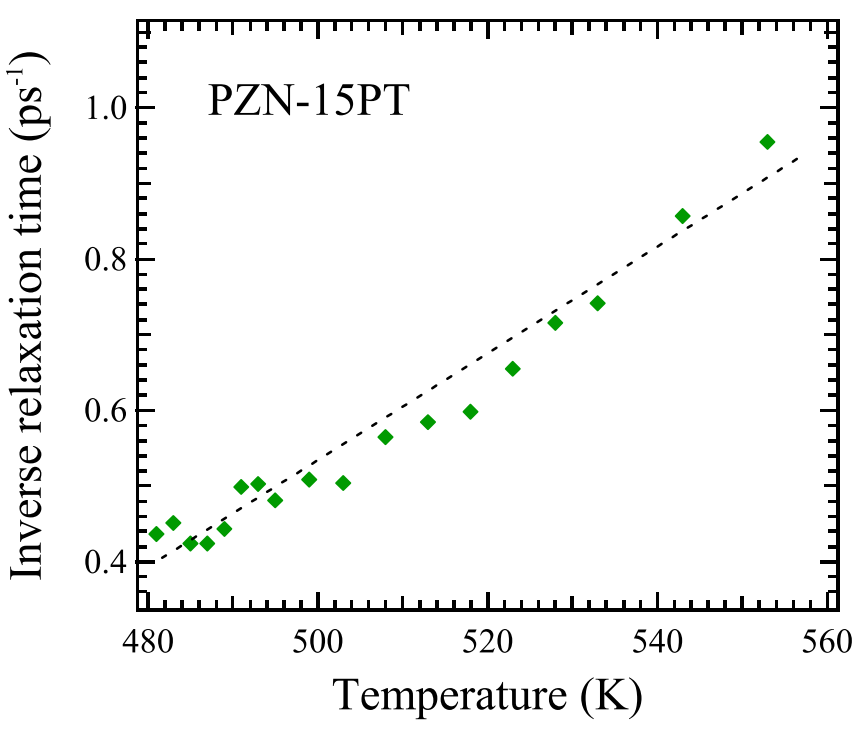

FIG. 4. Temperature dependence of the inverse relaxation time of a PZN15PT single crystal.

slowing down, which is the typical behavior of an orderdisorder phase transition.

\section{Critical slowing down}

Under the assumption of a single relaxation process, $\mathrm{CP}$ is fitted by using a single Lorentzian function centered at zero frequency shifts, and the relaxation time $\tau_{C P}$ is calculated by $\pi$. (FWHM of CP) $\times \tau_{\mathrm{CP}}=1$. The temperature dependence of the inverse of $\tau_{C P}$ is plotted in Fig. 4. A critical slowing down is clearly observed above $T_{C-T}=481 \mathrm{~K}$, which can be described by Eq. (1)

$$
\frac{1}{\tau}=\frac{1}{\tau_{0}}+\frac{T-T_{C}}{\tau_{1} T_{C}}, \quad \text { for } \quad T>T_{C-T},
$$

where $\tau_{0}=2.5 \mathrm{ps}$ and $\tau_{l}=0.30 \mathrm{ps}$. The value of $\tau_{1}$ is comparable with that of $0.70 \mathrm{~Pb}\left(\mathrm{Sc}_{1 / 2} \mathrm{Nb}_{1 / 2}\right) \mathrm{O}_{3}-0.30 \mathrm{PbTiO}_{3}$ ( $\mathrm{PSN}$ 30PT), while the value of $\tau_{0}$ is much smaller than that of PSN-30PT reflecting the first-order nature of the ferroelectric phase transition in PZN-15PT. ${ }^{16}$

The observed critical slowing down is characteristic of an order-disorder phase transition. In PZN-7PT, slowing down was observed; however, the critical slowing down is suppressed near $T_{\mathrm{C}}$ due to the existence of CORs. ${ }^{21,32} \mathrm{Simi-}$ lar phenomena were reported in PMN- $x \mathrm{PT} .{ }^{15}$ In PMN- $x \mathrm{PT}$, a strong spatial correlation between PNRs and nanoscale regions of chemical ordering (or CORs) on the B-site was suggested by recent first principle calculations. ${ }^{33}$ However, in PMN- $x$ PT, the size of CORs decreases with the increase of Ti-content and CORs finally disappear at $x \sim 0.4$, as revealed by transmission electron microscopy ${ }^{34}$ and synchrotron $\mathrm{x}$-ray scattering studies. ${ }^{35}$ Therefore, in PMN-55PT, the critical slowing down is clearly observed by Brillouin scattering. ${ }^{15}$ The dynamic behavior observed in PZN- $x$ PT can be explained in a similar way. In PZN- $x$ PT of low PT content, such as PZN-7PT, both the PNRs and the CORs could coherently contribute to the phase transition dynamics, and the critical slowing down is suppressed due to the existence of 


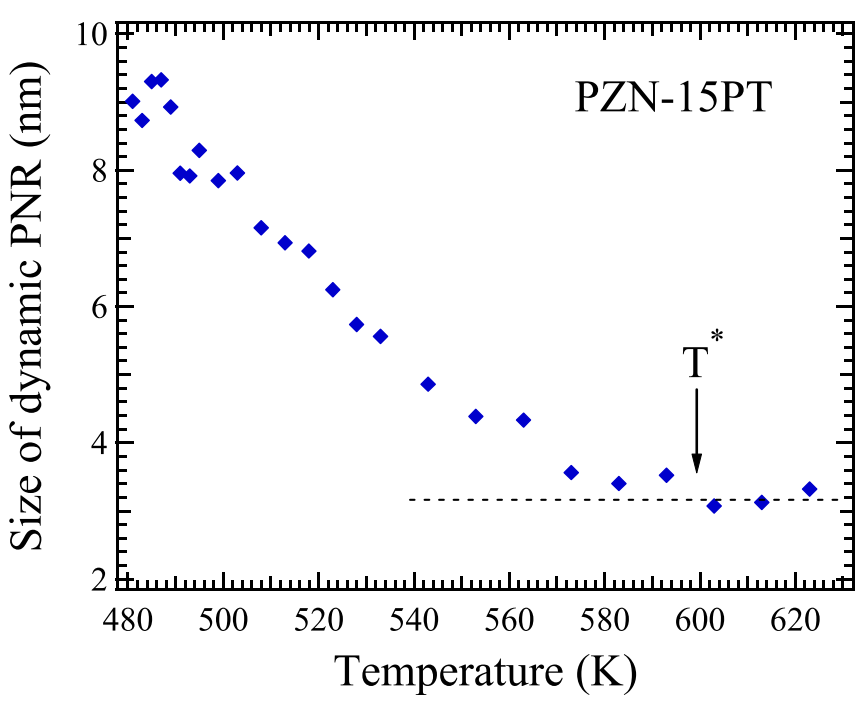

FIG. 5. Temperature dependence of the size of dynamic PNRs of a PZN15PT single crystal.

CORs. In contrast, in PZN-15PT, the CORs disappears or become very small with a higher PT-content, and only the polarization fluctuations in dynamic PNRs play a dominant role on the critical slowing down.

The growth and temperature evolution of the dynamic PNRs are also important in understanding the dynamics of PNRs of REFs. According to neutron diffuse scattering study, for PZN, a diffuse intensity peak appears at $T_{\mathrm{B}} \sim 720 \mathrm{~K}$ and the formation of static PNRs starts at temperature $T^{*} \sim 450 \mathrm{~K}^{36}$ and similarly, $T^{*} \sim 500 \mathrm{~K}$ for PZN-4.5PT, and $T^{*} \sim 550 \mathrm{~K}$ for PZN-9PT. ${ }^{37}$ For PZN-4.5PT, $T_{\mathrm{B}} \sim 800 \mathrm{~K}$ and $T^{*} \sim 550 \mathrm{~K}$ were reported by Raman scattering study. ${ }^{38}$ For PZN-4.5PT, the correlation lengths determined from the width of diffuse scattering are about $4-18 \AA$ at $T_{\mathrm{B}} \sim 720 \mathrm{~K}$, $13-30 \AA$ at $T^{*} \sim 500 \mathrm{~K}$, and $51-91 \AA$ at $300 \mathrm{~K}$, respectively. ${ }^{37}$

\section{Size of dynamic PNR}

In the present Brillouin scattering study, the size of a dynamic PNR, $l_{P N R}$, is estimated from the relaxation time related to local polarization flipping therein and the phase velocity of the LA mode, because the characteristic length of polarization flipping can be given by the propagation length of local strain in the period of a relaxation time. ${ }^{16,32}$ Figure 5 shows the temperature dependence of $l_{P N R}$ for PZN-15PT between $T_{\mathrm{B}} \sim 700 \mathrm{~K}$ and $T^{*} \sim 600 \mathrm{~K}$. At high temperatures, the size of dynamic PNRs is about $3 \mathrm{~nm}$ and remains nearly constant, while upon cooling below $T^{*}$ starts to increase sharply and reaches $13 \mathrm{~nm}$ near $T_{\mathrm{C}-\mathrm{T}}$ on cooling. These values are comparable with those of PZN-4.5PT determined by neutron diffuse scattering and the initial size of dynamic PNRs of $\sim 2.5 \mathrm{~nm}$ in PZN-7PT at $T^{*}$ determined by Brillouin scattering. ${ }^{16}$

\section{CONCLUSION}

In conclusion, we have shown the precursor dynamics of relaxor ferroelectric $0.85 \mathrm{~Pb}\left(\mathrm{Zn}_{1 / 3} \mathrm{Nb}_{2 / 3}\right) \mathrm{O}_{3}-0.15 \mathrm{PbTiO}_{3}$ single crystals using Brillouin and Raman scattering. The remarkable elastic softening and the increase of hypersonic damping were observed around $T_{C-T}$ demonstrating a strong interaction between the LA mode and the dynamic PNRs below $T_{\mathrm{B}}$. The local symmetry breaking related to PNRs was observed above $T_{C-T}$ by Raman scattering. The relaxation time estimated from the $\mathrm{CP}$ width shows a critical slowing down behavior above $T_{C-T}$, reflecting the absence of CORs at a higher concentration of PT. The size of dynamic PNR is found to increase markedly upon cooling down to $T_{\mathrm{C}-\mathrm{T}}$.

\section{ACKNOWLEDGMENTS}

The authors thank S. G. Lushnikov, J.-H. Ko, A. Bokov, G. Shabbir, and V. Sivasbrumanian for fruitful discussions. M.S.I. is grateful for accepting as a short-term visiting research fellow to University of Tsukuba and for the assistance of experiment of Brillouin scattering to T. H. Kim. The work at SFU was supported by the U.S. Office of Naval Research (Grants No. N00014-06-1-0166 and N00014-11-10552) and Natural Science and Engineering Research Council of Canada (NSERC).

${ }^{1}$ R. Blinc, Advanced Ferroelectricity, International Series of Monographs in Solid State Physics (Oxford University Press, 2011), Vol. 151.

${ }^{2}$ A. A. Bokov and Z.-G. Ye, J. Mater. Sci. 41, 31 (2006).

${ }^{3}$ S. Kojima, M. Ahart, V. Sivasbrumanian, A. A. Bokov, and Z.-G. Ye, J. Adv. Dielectr. 2(2), 1241004 (2012).

${ }^{4}$ D. E. Cox, B. Noheda, G. Shirane, Y. Uesu, K. Fujishiro, and Y. Yamada, Appl. Phys. Lett. 79, 400 (2001)

${ }^{5}$ Z. Kutnjak, J. Petzelt, and R. Blinc, Nature 441, 956 (2006).

${ }^{6}$ H. X. Fu and R. E. Cohen, Nature 403, 281 (2000).

${ }^{7}$ G. Xu, H. Hiraka, G. Shirane, and K. Ohwada, Appl. Phys. Lett. 84, 3975 (2004)

${ }^{8}$ G. Xu, D. Viehland, J. F. Li, P. M. Gehring, and G. Shirane, Phys. Rev. B 68, 212410 (2003)

${ }^{9}$ T. H. Kim, J.-H. Ko, S. Kojima, A. A. Bokov, X. Long, and Z.-G. Ye, Appl. Phys. Lett. 100, 082903 (2012).

${ }^{10}$ D. Phelan, X. Long, Y. Xie, Z.-G. Ye, A. M. Glazer, H. Yokota, P. A. Thomas, and P. M. Gehring, Phys. Rev. Lett. 105, 207601 (2010).

${ }^{11}$ E. Buixaderas, D. Nuzhnyy, J. Petzelt, L. Jin, and D. Damjanovic, Phys. Rev. B 84, 184302 (2011).

${ }^{12}$ A. A. Bokov, B. J. Rodriguez, X. Zhao, J.-H. Ko, S. Jesse, X. Long, W. Qu, T. H. Kim, J. D. Budai, A. N. Morozovska, S. Kojima, X. Tan, S. V. Kalinin, and Z.-G. Ye, Z. Kristallogr. 226, 99 (2011).

${ }^{13}$ G. Burns and F. H. Dacol, Phys. Rev. B 28, 2527 (1983).

${ }^{14}$ M. Roth, E. Mojaev, E. Dul'kin, P. Gemeiner, and B. Dkhil, Phys. Rev. Lett. 98, 265701 (2007).

${ }^{15}$ J.-H. Ko, S. Kojima, A. A. Bokov, and Z.-G. Ye, Appl. Phys. Lett. 91, 252909 (2007).

${ }^{16}$ S. Kojima, S. Tsukada, Y. Hidaka, A. A. Bokov, and Z.-G. Ye, J. Appl. Phys. 109, 084114 (2011).

${ }^{17}$ M. H. Kuok, S. C. Ng, H. J. Fan, M. Iwata, and Y. Ishibashi, Appl. Phys. Lett. 78, 1727 (2001).

${ }^{18}$ Y. Gorouya, Y. Tsujimi, M. Iwata, and T. Yagi, Appl. Phys. Lett. 83, 1358 (2003)

${ }^{19}$ D. H. Kim, J.-H. Ko, C. D. Feng, and S. Kojima, J. Appl. Phys. 98, 044106 (2005).

${ }^{20}$ D. H. Kim, S. Kojima, and J.-H. Ko, Curr. Appl. Phys. 11, S167 (2011).

${ }^{21}$ S. Tsukada and S. Kojima, Phys. Rev. B. 78, 144106 (2008).

${ }^{22}$ D. H. Kim, S. Kojima, and J.-H. Ko, J. Korean Phys. Soc. 46, 131 (2005).

${ }^{23}$ D. H. Kim, J.-H. Ko, C. D. Feng, and S. Kojima, Appl. Phys. Lett. 87, 072908 (2005).

${ }^{24}$ J.-H. Ko, D. H. Kim, and S. Kojima, Appl. Phys. Lett. 83, 2037 (2003).

${ }^{25}$ M. H. Kuok, S. C. Ng, H. J. Fan, M. Iwata, and Y. Ishibashi, Solid State Commun. 118, 169 (2001).

${ }^{26}$ M. Iwata, H. Hoshino, H. Orihara, H. Ohwa, N. Yasuda, and Y. Ishibashi, Jpn. J. Appl. Phys., Part 1 39, 5691 (2000).

${ }^{27}$ W. Chen and Z.-G. Ye, J. Cryst. Growth 233, 503 (2001).

${ }^{28}$ J.-H. Ko, D. H. Kim, S. Tsukada, S. Kojima, A. A. Bokov, and Z.-G. Ye, Phys. Rev. B 82, 104110 (2010). 
${ }^{29}$ F. Jiang and S. Kojima, Jpn. J. Appl. Phys., Part 1 38, 5132 (1999).

${ }^{30}$ D. La-Orauttapong, B. Noheda, Z.-G. Ye, P. M. Gehring, J. Toulouse, D. E. Cox, and G. Shirane, Phys. Rev. B 65, 144101 (2002).

${ }^{31}$ G. Burns and B. A. Scott, Phys. Rev. Lett. 25, 167 (1970).

${ }^{32}$ S. Kojima and S. Tsukada, Ferroelectrics 405, 32 (2010).

${ }^{33}$ S. Tinte, B. P. Burton, E. Cockayne, and U. V. Waghmare, Phys. Rev. Lett. 97, 137601 (2006).

${ }^{34}$ A. D. Hilton, D. J. Barber, C. A. Randall, and T. R. Shrout, J. Mater. Sci. 25, 3461 (1990).
${ }^{35}$ A. Tkachuk, P. Zschack, E. Colla, and H. Chen, Fundamental Physics of Ferroelectrics (American Institute of Physics, New York, 2001), Vol. 582, p. 45.

${ }^{36}$ D. La-Orauttapong, J. Toulouse, J. L. Robertson, and Z.-G. Ye, Phys. Rev. B 64, 212101 (2001).

${ }^{37}$ D. La-Orauttapong, J. Toulouse, Z.-G. Ye, W. Chen, R. Erwin, and J. L. Roberston, Phys. Rev. B 67, 134110 (2003).

${ }^{38}$ O. Svitelskiy, D. La-Orauttapong, J. Toulouse, W. Chen, and Z.-G. Ye, Phys. Rev. B 72, 172106 (2005). 